\title{
Mitosis and growth in biological tissues
}

\author{
J. C. M. Mombach, Rita M. C. de Almeida, and J. R. Iglesias \\ Instituto de Física, Universidade Federal do Rio Grande do Sul, \\ Caixa Postal 15051, 91501-970 Porto Alegre, Rio Grande do Sul, Brazil
}

(Received 5 November 1992)

\begin{abstract}
We present a simulation of the growth of a two-dimensional biological cellular system in which the cells experience mitosis whenever the (area)/(perimeter) ratio reaches a critical value. The model also includes the effect of interfacial energy and temperature. A stationary state with a constant average area is attained. We calculate the distribution of cells as a function of area, perimeter, and number of sides and also the two-cell correlation function. The results depend on temperature and are in agreement with experimental data, simulations, and theoretical models.
\end{abstract}

PACS number(s): 87.10.+e, 82.70.Rr

\section{INTRODUCTION}

Two-dimensional cellular systems like soap froths, sections of polycrystalline grains, or biological tissues present similar features in spite of originating through totally different phenomena [1]. This common behavior can be explained by noting that all these systems are subject to the same geometric and topological constraints: topology requires that every flat two-dimensional, spacefilling cellular structure has cells with an average of six sides and geometry imposes a relation between area and perimeter that is maximal for a regular polygon. Also, from a physical point of view, the main contribution to the energy comes from the interfaces between the cells.

The dynamics of soap froths and metallurgical grains are relatively well understood. The equilibrium distribution functions can be calculated, for instance, through the maximum-entropy formalism [2-4]. Nearly twodimensional biological tissues exhibit a distribution of cells that is similar to foams and metallurgical aggregates $[1,5]$ in spite of being generated by a different dynamics: biological cells may suffer mitosis, the process in which cells mature and split in two.

As was pointed out by Lewis [6] the surface tensions of the cells play an essential role in determining the overall pattern both of biological tissues and soap froths [7]. However, the long-time state of a biological tissue differs from foams in an important detail: the average area of the cells is constant in time. Unlike other systems, biological cells may suffer mitosis. While there has been some theoretical modeling of cellular growth in biological systems, another possible approach to study this problem is to perform a simulation that takes into account the main features of cellular growth. These simulations can then be compared with the results of experiments [5, $6]$ and theoretical predictions for two-dimensional cellular systems. The aim of this paper is to present such a numerical simulation of the growth of a two-dimensional cellular system on the same basis as recent simulations for cell sorting $[8,9]$, soap froths, and grain growth [10-13], but including cell mitosis. The results are then compared with experiments performed by two of us [5]. They are in good agreement with the experimental data and also with such general principles as the Aboav-Weaire law [1, $14,15]$.

\section{SIMULATION PROCEDURE}

The simulation is performed on a square lattice where a positive integer label is assigned to each site: a connected set of equally labeled sites represents a cell. The entire lattice contains $1100 \times 1100$ sites. Sites labeled with 0 are empty sites. Cells can grow either by invading 0 sites, in which case we assume that there is no interfacial energy, or by invading neighboring cells' sites, thus modifying the interfacial energy which we take to be proportional to the length of the contact region.

The simulation begins with an initial cell (labeled 1) of $10 \times 10$ sites at the center of the lattice. As mentioned above, there is no interfacial energy between the cell and the vacuum ( 0 sites) and two sites with different indices contribute with a fixed value $\delta$ to the energy if they are nearest neighbors. The cells grow as follows: (i) Choose at random a site and one of its neighbors. (ii) If both sites belong to the same cell, return to (i). (iii) The probability that a site is invaded, that is, the probability of changing the site label to the label of the neighbor is given by

$$
P(\Delta E)=\left\{\begin{array}{cl}
\exp (-\beta \Delta E) & \text { if } \Delta E>0 \\
1 & \text { if } \Delta E \leq 0
\end{array}\right.
$$

where $\beta$ is associated with the "thermal" noise and $\Delta E$ is the energy difference between final and initial states.

This is not the only mechanism to modify the tissue. The cells also suffer mitosis when they attain a critical value of the (area)/(perimeter) ratio [16]. Here area is measured by the number of equally labeled sites and perimeter by the number of lattice edges that separate 
two cells. Mitosis results from a competition between surface and bulk-energy terms similar to the origin of finite domains in ferromagnetic materials. In biological tissues the competition arises from the capacity of the cellular membrane to absorb nutrients (proportional to the perimeter) versus the required amount of these nutrients to keep the cell alive, which is proportional to the volume of the cell (proportional to the area, in two-dimensional tissues). The mitosis process is then responsible for a constant average area of the cell that is related to an optimal (area)/(perimeter) ratio, which may be different for different biological systems. Consequently, choosing the critical value of the ratio that determines the onset of mitosis is equivalent to defining the average area of the cells.

In the simulation the mitosis is performed as follows: periodically in the program a mitosis routine is run: the center of mass, area $a$, and perimeter $p$ of each cell are measured and whenever the ratio $a / p$ exceeds a given mitosis parameter $k$ (here $k=3$ ), the cell suffers mitosis, i.e., it is divided into two different cells by an edge crossing the center of mass in the direction of the least diameter, as occurs in real tissues [16]. Also, during the growth process a cell may split into two or more pieces. Then from time to time a routine is run to locate all disconnected pieces of a given cell and attribute different labels. This procedure guarantees that cells are simply connected at all temperatures.

Finally, we have performed the simulation for different values of the temperature $T=1 / \beta$ from 0 to 0.9 and the results are presented in the following section.

\section{RESULTS}

The simulation is performed on a lattice of $1100 \times 1100$ sites, but we monitored the evolution of a region with $300 \times 300$ sites at the center of the lattice, to avoid surface effects. The simulation ends when the growing tissue touches the far edges of the lattice. We chose a critical value of 3 for the (area)/(perimeter) ratio to ensure that in the stationary regime there are enough sites per cell and enough cells in the monitored region of the tissue to guarantee statistically significant results. In Fig. 1 we show six snapshots of a region of $100 \times 100$ sites of the central tissue, grown at different temperatures. At $T=0$ the process is fully deterministic and the tissue presents irregular cells: although most of the sides are straight in either the horizontal or vertical directions, in order to minimize the wall energy, as it is to be expected on a square lattice with first-neighbors interactions. This effect is more evident for $T=0.1$ and 0.2 : it is a "pinning" effect [17], also present in previous simulations [11, 13], originating from the anisotropy of the square lattice. Thermal energy overcomes pinning effects for $T \geq 0.3$ and for $T=0.6$ and 0.9 the tissue is disordered and with more "rounded" cells. We have chosen the tissue grown at $T=0.6$ to investigate the statistical properties of the system. For all temperatures we averaged over 5 runs except for $T=0.6$, where we considered 10 simulated tissues.

First of all we must be sure that the system has at-

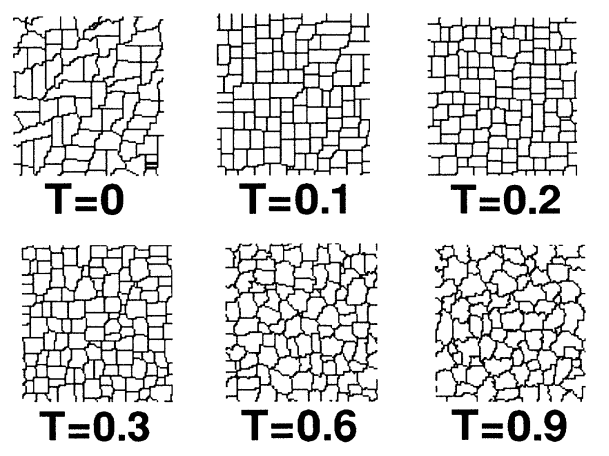

FIG. 1. Snapshots of tissues grown at different temperatures.

tained an equilibrium configuration. In Fig. 2(a) we have plotted the mean area of the cells as a function of the number of Monte Carlo simulation steps per site, MCS. It is evident that after 400 MCS the tissue attains a constant average area, as expected in biological tissues. This constant area is ensured by mitosis; in other cellular systems, like soap froths and metallurgical aggregates where mitosis is not possible, the average area increases as a power law in time, although the structure remains selfsimilar $[1,18,19]$. In Fig. 2(b) we plot the second moment of the distribution of number of sides, $\mu_{2}$, a parameter that is widely used as a measure of the disorder of the lattice; in both figures it is evident that the tissue attains an equilibrium regime after about $500 \mathrm{MCS}$, with $\mu_{2} \approx 1.7$.

\section{A. Disorder of the tissue}

In Fig. 3 we plot the probability of finding a cell with $n$ sides for tissues grown at $T=0.6$ for three different times. The mean value is roughly 6 , as expected, and the dispersion $\mu_{2}=1.71$, while real biological systems exhibit $\mu_{2} \approx 1$. They are more regular than both the present simulation as well as two-dimensional soap froths, if one compares with the experimental results of Glazier, Anderson, and Grest [10]. The value of $\mu_{2}$ depends on the temperature of our simulations. As shown in Fig. 4(a)
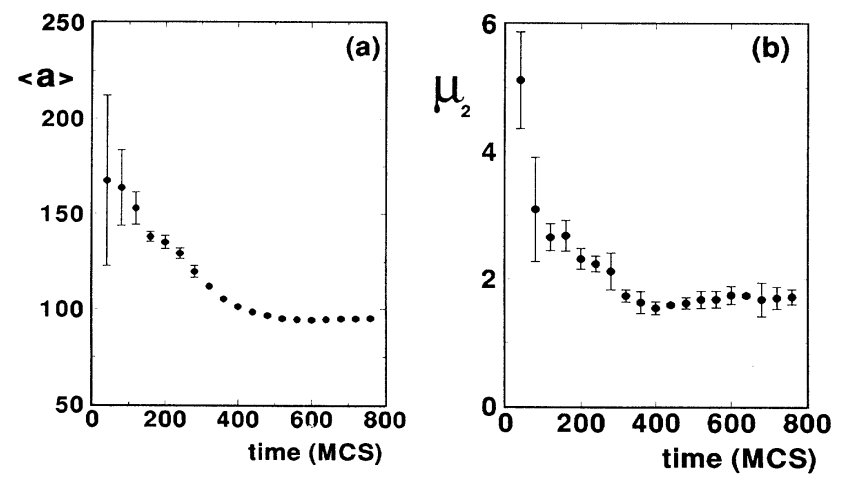

FIG. 2. (a) Average area as a function of time. (b) Temporal evolution of the disorder parameter $\mu_{2}=\left\langle(n-6)^{2}\right\rangle$. Here MCS stands for Monte Carlo step per site. 


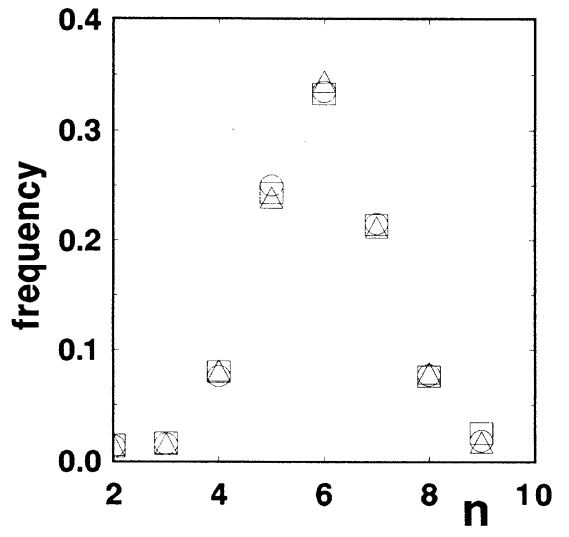

FIG. 3. Plot of the distribution of sides for $t=500, t=$ 600 , and $t=700 \mathrm{MCS}$, all in the stationary state.

$\mu_{2}$ is very large (and with a large error bar) for $T=0$, where the tissue is frozen in a metastable state, decreases to very low values for $T=0.1$ and 0.3 due to the pinning effect, and slowly increases with $T$ after that. This behavior is also clear from the plot of the mean area $\langle a\rangle$ as a function of $T$, but a different derivative is obtained for $T>0.6$, Fig. 4(b). As the pinning "freezes" the tissue at low temperatures, one must perform the simulation at relatively high temperatures $(T \approx 0.6)$ and so the simulated tissue is more disordered than real biological ones. We expect that a simulation performed on lattices where the energy anisotropy is lower and pinning effects not relevant [13] should improve these results and enable a better quantitative comparison with experiments.

To determine the size of the cells we plot the distribution of area of the cells (normalized to the mean value $\langle a\rangle)$, as shown in Fig. 5 for three different times, at $T=0.6$. Note that all simulation points lie in the same region while the experimental data [6] (full triangles) exhibit a lower dispersion, as occurs for the distribution in the number of sides. We have also performed simulations on different samples and the results are the same.

\section{B. Lewis's law}

One interesting point is the relation between the mean area of $n$-sided cells $\left\langle a_{n}\right\rangle$ as a function of the number
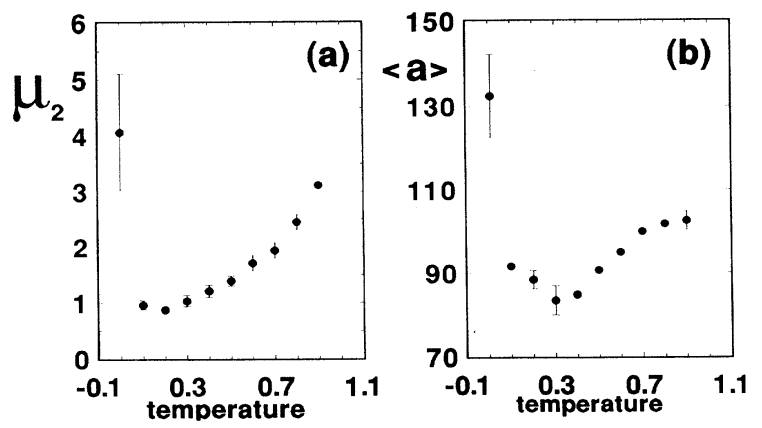

FIG. 4. Temperature dependence of (a) $\mu_{2}$ and (b) the average area of the cells $\langle a\rangle$.

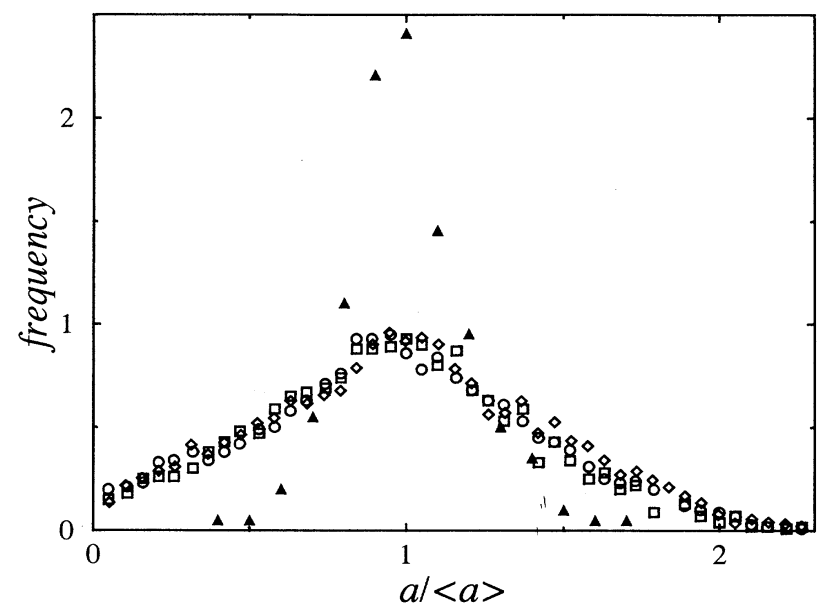

FIG. 5. Area distribution for three different instants of time in the stationary state (open symbols) compared with Lewis's experimental results for cucumber (full triangles) [6].
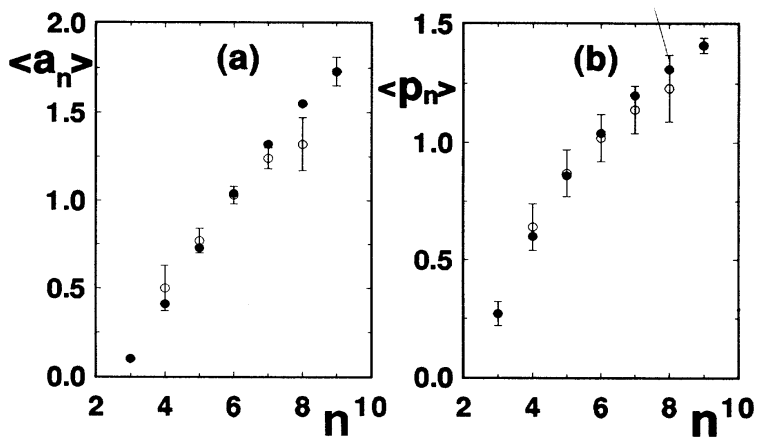

FIG. 6. (a) Average area and (b) perimeter of $n$-sided cells $\left\langle a_{n}\right\rangle$ for A. attenuata (open circles) [5] and our simulation results (full circles).
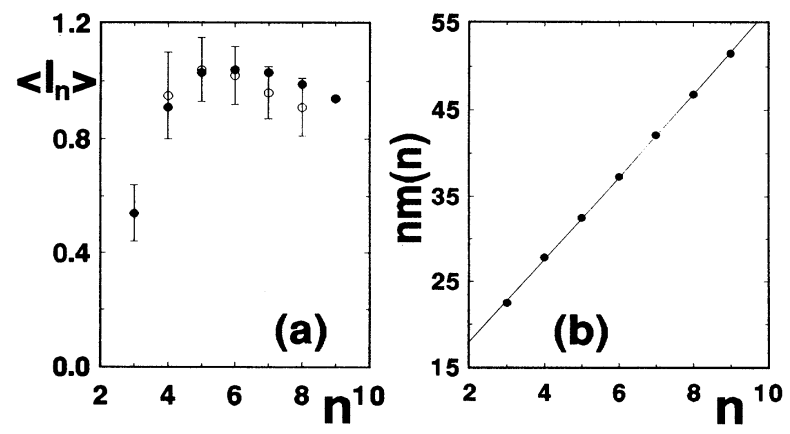

FIG. 7. (a) Average side length of the $n$-sided cells for $A$. attenuata (open circles) [5] and simulation results (full circles). (b) Verification of the Aboav-Weaire's law for the simulated tissues. The slope of the line is 4.8 . 


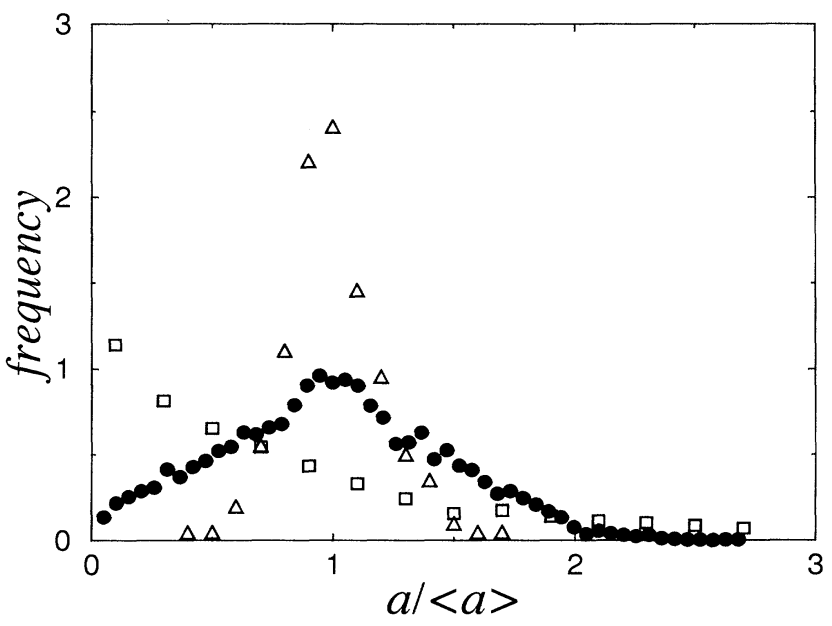

FIG. 8. Area distribution for (a) the Potts model (open squares) [10], cucumber (open triangles) [6], and (b) our simulation results (full circles).

of sides $n$. Lewis [16] found that the relation between $\left\langle a_{n}\right\rangle$ and $n$ is approximately linear, i.e., $\left\langle a_{n}\right\rangle \approx A+B n$. Some maximum entropy theoretical results also predict a linear relationship [2], others give a different exponent $\left(\left\langle a_{n}\right\rangle \approx n^{2}\right)[4]$, and the random-neighbor model obtains the linear relationship as an asymptotic law [20]. Unfortunately experimental results are not conclusive [5]. In Fig. 6(a) we have plotted the results of our simulation together with experimental results for A. attenuata [5] and both seem to indicate a linear dependence on $n$ up to $n=9$, the maximum number of sides obtained in the simulation. In Fig. 6(b) we have plotted the results of the simulation and experimental data for the mean perimeter and in Fig. 7(a) the average side length of $n$-sided cells. Although it is not trivial to determine the functional relationship, both results are in very good agreement with the experiments.

\section{Aboav-Weaire's law}

The relation between the number of sides of a cell and the mean number of sides of its neighbors $m(n)$ deserves special attention. An empirical universality appears in experiments and numerical simulations $[1,5,10,21,22]$. It has been observed that

$$
n m(n)=(6-a) n+\left(6 a+\mu_{2}\right)
$$

where $a$ is always close to 1 , yielding a slope near 5 in the plot $n m(n)$ vs $n$. A detailed discussion of the probable origins of the Aboav-Weaire's law as well as its verification in many different cases was presented elsewhere [23]. Here we restrict ourselves to a plot of $n m(n)$ vs $n$, Fig. 7(b), where we show that our simulation also verifies Eq. (2). This is in agreement with experimental results for soap froths [15], vegetable tissues [5], and with earlier simulations $[10,21]$.
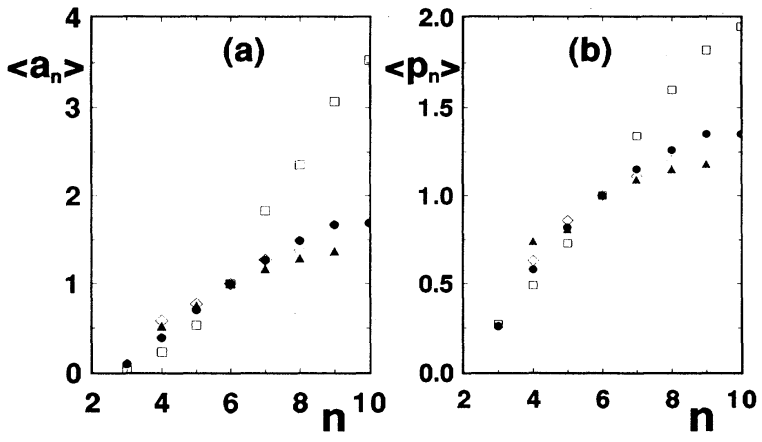

FIG. 9. Average area $\left\langle a_{n}\right\rangle$ (a) and perimeter $\left\langle p_{n}\right\rangle$ (b) of the $n$-sided cells for the Potts model (open squares) [10], $A$. arborescens (diamonds) [5], cf. Anthurium (full triangles) [5], and our simulation results (full circles).

\section{DISCUSSION AND CONCLUSIONS}

The main difference between biological tissues and other cellular systems is the ability of biological cells to split themselves: mitosis. This guarantees a long-time stable state and a constant average area (volume, in three dimensions). We have shown that a simple simulation including mitosis can reproduce important features [5] of two-dimensional biological tissues. In order to compare with other simulations without mitosis we have plotted in Fig. 8 the area distributions obtained in a Potts-model simulation [10] and in the experiments by Lewis [6], together with our results. It is evident that simulations without mitosis yield a distribution with a maximum for zero-area cells, while we found a maximum for finite area, although with a larger dispersion (due to the anisotropy [13]) than Lewis's experimental results. Finally the plot of the average area of $n$-sided cells $\left\langle a_{n}\right\rangle$ and the average perimeter $\left\langle p_{n}\right\rangle$ displays a very good agreement between the present simulation and experimental data. For comparison we also plotted in Figs. 9(a) and 9(b) the Potts model simulation data for the average area and radius of $n$-sided cells, respectively: the results diverge for $n>6$.

These results suggest that mitosis is the dynamical process that accounts for the differences between metallurgical aggregates or soap froth and biological cells [5]. Simulations on a triangular lattice, where pinning effects are not relevant, are now in progress and should improve further quantitative agreement with experiments.

\section{ACKNOWLEDGMENTS}

We acknowledge fruitful discussions with M. A. Gusmão, P. M. Mors, N. Lemke, and J. J. Arenzon. This work has been partially supported by Brazilian agencies $\mathrm{CNPq}$ (Conselho Nacional de Desenvolvimento Científico e Tecnológico), FINEP (Financiadora de Estudos e Projetos), and FAPERGS (Fundação de Amparo à Pesquisa do Estado do Rio Grande do Sul). 
[1] D. Weaire and N. Rivier, Contemp. Phys. 25, 49 (1984).

[2] N. Rivier, Philos. Mag. B 52, 795 (1985).

[3] M.A. Peshkin, K.J. Strandburg, and N. Rivier, Phys. Rev. Lett. 67, 1803 (1991).

[4] J.R. Iglesias and R.M.C. de Almeida, Phys. Rev. A 43, 2763 (1991).

[5] J.C.M. Mombach, M.A.Z. Vasconcellos, and R.M.C. de Almeida, J. Phys. D 23, 600 (1990).

[6] F.T. Lewis, Anat. Rec. 50, 235 (1931).

[7] Sir D. Thompson, On Growth and Form, 2nd ed. (Cambridge University Press, Cambridge, 1942).

[8] F. Graner and J.A. Glazier, Phys. Rev. Lett. 69, 2013 (1992).

[9] J.A. Glazier and F. Graner, Phys. Rev. E 47, 2128 (1993).

[10] J.A. Glazier, M.P. Anderson, and G.S. Grest, Philos. Mag. B 62, 615 (1990).

[11] M.P. Anderson, D.J. Srolovitz, G.S. Grest, and P.S.
Sahni, Acta Metall. 32, 783 (1984).

[12] D.J. Srolovitz, M.P. Anderson, P.S. Sahni, and G.S. Grest, Acta Metall. 32, 793 (1984).

[13] E.A. Holm, J.A. Glazier, D.J. Srolovitz, and G. S. Grest, Phys. Rev. A 43, 2662 (1991).

[14] D.A. Aboav, Metallography 13, 43 (1980).

[15] D.A. Aboav, Metallography 16, 265 (1983).

[16] F.T. Lewis, Anat. Rec. 38, 341 (1928).

[17] J. Viñals and M. Grant, Phys. Rev. B 36, 7036 (1987).

[18] J. Stavans and J.A. Glazier, Phys. Rev. Lett. 62, 1318 (1989)

[19] J.A. Glazier and D. Weaire, J. Phys. C 4, 1867 (1992).

[20] H. Flyvbjerg, Phys. Rev. E 47, 4037 (1993).

[21] D. Weaire and J.P. Kermode, Philos. Mag. B 48, 245 (1983).

[22] G. Le Caër, J. Phys. A 24, 1307 (1991).

[23] J.C.M. Mombach, R.M.C. de Almeida, and J. R. Iglesias, Phys. Rev. E 47, 3712 (1993). 